\title{
Modelos lineares e não lineares inteiros para problemas da mochila bidimensional restrita a 2 estágios
}

\author{
Horacio Hideki Yanasse ${ }^{\mathrm{a} *}$, Reinaldo Morabito ${ }^{\mathrm{b}}$ \\ a*horacio@lac.inpe.br, INPE, Brasil \\ bmorabito@ufscar.br, UFSCar, Brasil
}

\begin{abstract}
Resumo
Neste trabalho revemos alguns modelos lineares e não lineares inteiros para gerar padrões de corte bidimensionais guilhotinados de 2 estágios, incluindo os casos exato e não exato e restrito e irrestrito. Esses problemas são casos particulares do problema da mochila bidimensional. Apresentamos também novos modelos para gerar esses padrões de corte, baseados em adaptações ou extensões de modelos para gerar padrões de corte bidimensionais restritos 1-grupo. Padrões 2 estágios aparecem em diferentes processos de corte, como, por exemplo, em indústrias de móveis e de chapas de madeira. Os modelos são úteis para a pesquisa e o desenvolvimento de métodos de solução mais eficientes, explorando estruturas particulares, a decomposição do modelo, relaxações do modelo etc. Eles também são úteis para a avaliação do desempenho de heurísticas, já que permitem (pelo menos para problemas de tamanho moderado) uma estimativa do gap de otimalidade de soluções obtidas por heurísticas. Para ilustrar a aplicação dos modelos, analisamos os resultados de alguns experimentos computacionais com exemplos da literatura e outros gerados aleatoriamente. Os resultados foram produzidos usando um software comercial conhecido e mostram que o esforço computacional necessário para resolver os modelos pode ser bastante diferente.
\end{abstract}

Palavras-chave

Problemas de corte e empacotamento. Mochila bidimensional. Corte guilhotinado-2 estágios. Modelos lineares e não lineares inteiros. Indústria de móveis.

\section{Introdução}

Problemas de corte aparecem em muitos processos industriais em que rolos de papel e alumínio, chapas de vidro e fibra de vidro, barras e chapas de metais e madeira, pedaços de tecido ou de couro etc. são cortados para produzir uma certa quantidade de peças menores demandadas. 0 problema é determinar a melhor maneira de cortar os objetos maiores para produzir os itens demandados de modo a otimizar um ou mais objetivos, por exemplo, minimizar perdas de material. Para revisões e edições especiais em problemas de corte e empacotamento e suas aplicações industriais, os leitores podem consultar Dyckhoff e Waescher (1990), Lirov (1992), Dowsland e Dowsland (1992), Sweeney e Paternoster (1992), Dyckhoff e Finke (1992), Martello (1994a, b), Bischoff e Waescher (1995), Mukhacheva (1997), Dyckhoff, Scheithauer e
Terno (1997), Arenales, Morabito e Yanasse (1999), Wang e Waescher (2002), Hifi (2002), Lodi, Martello e Monaci (2002), Waescher, Haussner e Schumann (2007), Morabito, Arenales e Yanasse (2009), Kendall, Daniels e Burke (2010) e ESICUP (EURO..., 2011).

Em alguns processos de corte, como na indústria de móveis e de chapas de madeira, o equipamento de corte é capaz de produzir apenas cortes guilhotinados. Um corte é guilhotinado se vai de um lado até o outro da chapa sem mudança de direção. Um corte é do tipo ortogonal guilhotinado se, aplicado a uma chapa retangular, produz dois novos retângulos. Gilmore e Gomory (1965) apresentam um método efetivo para lidar com esse problema quando o padrão de corte é do tipo guilhotinado com no máximo 2 estágios. No primeiro estágio, cortes longitudinais paralelos 
são produzidos na chapa, sem movê-la, produzindo um conjunto de tiras (faixas). No segundo estágio, essas tiras são empurradas, uma a uma, e os cortes transversais restantes são feitos em cada tira (Figura 1). Se não existe necessidade de uma apara adicional (i.e., todas as peças têm a mesma largura em cada tira), o padrão é dito ser guilhotinado 2-estágios exato (Figura 1a); caso contrário, é chamado guilhotinado 2-estágios não exato (Figura 1b). Outros estudos que abordam o problema de corte na indústria de móveis e de chapas de madeira podem ser encontrados em Gilmore e Gomory (1965), Foronda e Carino (1991), Yanasse, Zinober e Harris (1991), Carnieri, Guillermo e Gavinho (1994), Morabito e Garcia (1998), Morabito e Arenales (2000), Vianna, Arenales e Gramani (2003), Gramani e França (2006), Morabito e Belluzzo (2007), Rangel, Figueiredo e Altamiro (2008), Gramani, França e Arenales (2009), Macedo, Alves e Carvalho (2010), Silva, Alvelos e Carvalho (2010), Santos, Araujo e Rangel (2011) e Alem e Morabito (2012). Em particular, Macedo, Alves e Carvalho (2010) e Silva, Alvelos e Carvalho (2010) exploraram abordagens alternativas ao método desenvolvido por Gilmore e Gomory (1965), baseadas em modelos arco-fluxo de programação linear inteira e em extensões do modelo um-corte de programação linear inteira de Dyckhoff (1981), respectivamente.

0 método de Gilmore e Gomory é baseado no método simplex com um procedimento de geração de colunas que envolve a solução de um problema da mochila bidimensional para gerar padrões 2-estágios. Esse procedimento envolve duas fases: na primeira fase, padrões de corte são determinados para cada tira longitudinal. Na segunda fase, decide-se quantas vezes cada tira deve ser usada. 0 método de Gilmore e Gomory funciona bem quando as quantidades ordenadas das peças são "suficientemente" grandes; nestes casos, o problema de corte é denominado irrestrito. Abordagens baseadas em duas fases são comuns na literatura, como, por exemplo, em Farley (1983), Riehme, Scheithauer e Terno (1996), Hifi (1997), Morabito e Garcia (1998), Vianna et al. (2003) e Yanasse e Katsurayama (2005). Para o caso restrito em que as quantidades de peças são relativamente pequenas, autores têm proposto alternativas para as soluções relaxadas do método simplex (WAESCHER; GAU, 1996; POLDI; ARENALES, 2006), ou heurísticas gulosas combinadas com procedimentos de geração de colunas (HINXMAN, 1980; POLDI; ARENALES, 2009), que podem não funcionar bem em determinadas situações. Outros estudos que abordam padrões estagiados podem ser encontrados em Beasley (1985), Christofides e Hadjiconstantinou (1995), Morabito e Arenales (1996), Riehme, Scheithauer e Terno (1996), Hifi (1997), Hifi e Roucairol (2001),
Vianna et al. (2003), Lodi e Monaci (2003), Cui (2005), Belov e Scheithauer (2006), Cintra et al. (2008) e Morabito e Pureza (2010).

Devido a características particulares de certas máquinas de corte, classes especiais de padrões 2-estágios que requerem tempos menores de processamento também são utilizadas na indústria de móveis, como os padrões guilhotinados 1-grupo. Nesses padrões, os cortes do segundo estágio são realizados com todas as tiras obtidas do primeiro estágio de maneira simultânea (GILMORE; GOMORY, 1965; MORABITO; ARENALES, 2000; SCHEITHAUER, 2002; YANASSE; KATSURAYAMA, 2005; YANASSE; MORABITO, 2006). Isso implica que os cortes são feitos juntos no segundo estágio, sem movimentar as tiras, e dessa forma economizando tempo de processamento. Gilmore e Gomory (1965) e Yanasse e Morabito (2008) também discutem padrões $p$-grupo, $p>1$. Note que padrões 1-grupo podem ser não homogêneos (um padrão é homogêneo se contém apenas um único tipo de item) exatos e não exatos, dependendo da necessidade de aparas adicionais, como ilustrado na Figura 2.

Poucos trabalhos foram encontrados na literatura que apresentam formulações matemáticas para problemas de corte guilhotinados 2-estágios, vistos como casos particulares do problema da mochila bidimensional (WAESCHER; HAUSSNER; SCHUMANN, 2007). Modelos para tais problemas podem ser úteis para a pesquisa e desenvolvimento de métodos de solução mais efetivos, explorando

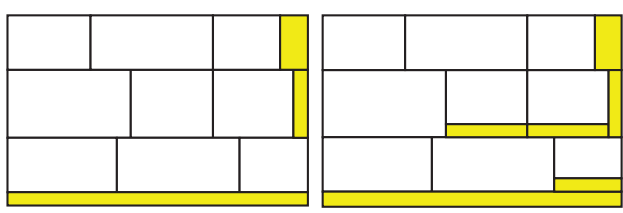

Figura 1. Padrões de corte 2-estágios: (a) caso exato, (b) caso não exato.

(a)

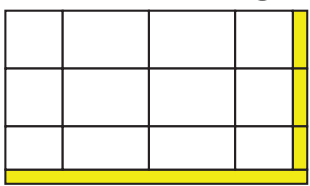

(b)

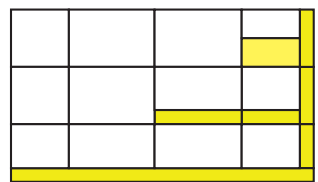

(c)

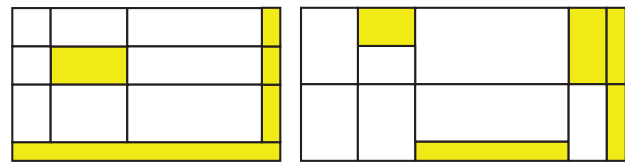

Figura 2. Padrões de corte 1-grupo: (a) e (c) casos exatos, (b) e (d) casos não exatos. 
características especiais e estruturas particulares, decomposição do modelo, relaxações do modelo etc. Esses modelos podem ser úteis também para a avaliação de desempenho de heurísticas, pois eles permitem (pelo menos para problemas de tamanho moderado) uma estimação do gap de otimalidade de suas soluções. Motivados por isso, neste estudo revemos modelos inteiros lineares e não lineares da literatura para gerar padrões 2-estágios e propomos novos modelos, incluindo os casos exato e não exato e restrito e irrestrito. Tais modelos também podem ser usados no procedimento de geração de colunas de Gilmore e Gomory, ou combinados com heurísticas de redução por repetição exaustiva (HINXMAN, 1980), para resolver problemas de corte de estoque. Além disso, eles podem ser combinados em formulações do problema bidimensional de empacotamento de bins (WAESCHER et al., 2007).

Este artigo está organizado da seguinte maneira: na seção 2, descrevemos brevemente os modelos lineares e não lineares existentes para o problema de corte 2-estágios. Na seção 3, propomos modelos lineares inteiros alternativos, alguns deles baseados na adaptação ou extensão de modelos de padrões de corte 1-grupo. Apesar da comparação efetiva dos modelos estar além do escopo deste trabalho, na seção 4, para efeito de ilustração, apresentamos os resultados de alguns experimentos computacionais da aplicação dos modelos para resolver alguns problemas exemplos da literatura e outros gerados de maneira aleatória. Utilizamos um software comercial bem conhecido de linguagem de modelagem GAMS e o resolvedor de programas lineares inteiros mistos CPLEX. Os resultados mostram que os esforços computacionais requeridos pelos modelos podem ser bastante diferentes, pelo menos para problemas de tamanho moderado. Finalmente, na seção 5, apresentamos as considerações finais e discutimos perspectivas de pesquisas futuras.

\section{Revisão de modelos de corte 2-estágios}

Nesta breve revisão admitimos, sem perda de generalidade, que os cortes do primeiro estágio são realizados paralelos ao comprimento da chapa, e os do segundo estágio são paralelos à largura da chapa. Admitimos também que a orientação das peças está fixada, ou seja, as peças não podem sofrer rotação. Sejam os seguintes parâmetros:

- $L, W \rightarrow$ comprimento e largura da chapa

- $m \rightarrow$ número total de tipos de peças

- $l_{i}, w_{i} \rightarrow$ comprimento e largura da peça tipo $i$ $(i=1, \ldots, m)$
- $l_{\min }, w_{\min } \rightarrow$ menor comprimento e menor largura, respectivamente, das peças

- $v_{i}, b_{i} \rightarrow$ valor (e.g., área) e demanda da peça tipo $i$, respectivamente.

Também sem perda de generalidade, admitimos que $b_{i} \leq\left\lfloor\left(L / l_{i}\right)\left(W / w_{i}\right)\right\rfloor$, para $i=1, \ldots, m$, uma vez que $\left\lfloor\left(L / I_{i}\right)\left(W / W_{i}\right)\right\rfloor$ é um limitante superior para o número máximo de peças tipo $i$ que podem ser cortados da chapa por um padrão 2-estágios guilhotinado. Dizemos que o problema é irrestrito se $b_{i}=\left\lfloor\left(L / l_{i}\right)\left(W / w_{i}\right)\right\rfloor$ para todo $i$; caso contrário é restrito.

\subsection{Modelos inteiros não lineares}

Vianna, Arenales e Gramani (2003) apresentaram o seguinte modelo inteiro não linear (VAG1) para o caso não exato. Sejam os parâmetros:

- $K \rightarrow$ número de larguras diferentes $w_{i}$.

- $P_{k} \rightarrow$ número máximo possível de tiras $L \times w_{k}$ (observe que $P_{k} \leq\left\lfloor W / w_{k}\right\rfloor$ )

- $I_{k}=\left\{i \mid w_{i} \leq w_{k}\right\}$

e as variáveis de decisão:

- $\lambda_{k p}^{i} \rightarrow$ número de vezes que a peça tipo $i$ é cortada do $p$-ésimo padrão da tira $L \times w_{k}$

- $\mu_{k p} \rightarrow$ número de vezes que o $p$-ésimo padrão da tira $L \times w_{k}$ é cortada ao longo da largura $W$ da chapa

$\max \sum_{k=1}^{K} \sum_{p=1}^{P_{k}} \sum_{i \in I_{k}} v_{i} \lambda_{k p}^{i} \mu_{k p}$

Sujeito a

$\sum_{i \in l_{k}} l_{i} \lambda_{k p}^{i} \leq L$, para $k=1, \ldots, K, p \in P_{k}$

$\sum_{k=1}^{K} w_{k} \sum_{p=1}^{P_{k}} \mu_{k p} \leq W$

$\sum_{k=1}^{K} \sum_{p=1}^{P_{k}} \lambda_{k p}^{i} \mu_{k p} \leq b_{i}$, para $i=1, \ldots, m$

com

$\lambda_{k p}^{i}, \mu_{k p} \geq 0$, inteiro, $i=1, \ldots, m ; k=1, \ldots, K ; p=1, \ldots, P_{k}$

A função objetivo (1) maximiza o valor total das peças cortadas no padrão. As restrições (2) impõem que o comprimento total das peças arranjadas nas tiras não exceda o comprimento da chapa. As restrições (3) impõem que a largura total das tiras arranjadas na placa não exceda a largura da chapa. As restrições (4) limitam o corte de peças a no máximo 
suas demandas. As restrições (5) referem-se à não negatividade e integralidade das variáveis. Note que sem as restrições (4), o modelo anterior corresponde ao caso do problema de corte 2-estágios irrestrito e ele, então, pode ser decomposto nos subproblemas explorados no método de duas fases de Gilmore e Gomory (1965), como mostrado em Vianna, Arenales e Gramani (2003).

0 número de peças tipo $i$ cortadas no padrão pode ser definido como:

$\alpha_{i}=\sum_{k=1}^{K} \sum_{p=1}^{P_{k}} \delta_{k}^{i} \lambda_{k p}^{i} \mu_{k p}$, em que $\delta_{k}^{i}= \begin{cases}1, & \text { se } i \in I_{k} \\ 0, & \text { caso contrário. }\end{cases}$

Substituindo essa equação no modelo (1)-(5), obtemos o modelo VAG2:

\section{Modelo VAG2}

$\max \sum_{i=1}^{m} v_{i} a_{i}$

Sujeito a

$\sum_{i \in l_{k}} l_{i} \lambda_{k p}^{i} \leq L$, para $k=1,, \ldots, K, p \in P_{k}$

$\sum_{k=1}^{K} w_{k} \sum_{p=1}^{P_{k}} \mu_{k p} \leq W$

$\alpha_{i}=\sum_{k=1}^{K} \sum_{p=1}^{P_{k}} \delta_{k p}^{i} \lambda_{k p}^{i} \mu_{k p}$, para $i=1, \ldots, m$

$\alpha_{i} \leq b_{i}$, para $i=1, \ldots, m$

com

$\lambda_{k p}^{i}, \mu_{k p} \geq 0$, inteiro, $i=1, \ldots, m$;

$k=1, \ldots, K ; p=1, \ldots, P_{k}$

Essa substituição tem o inconveniente de aumentar o número de variáveis e restrições, mas simplifica a apresentação da linearização apresentada a seguir. Observe que o caso de corte 2-estágios exato pode ser representado pelos modelos anteriores simplesmente redefinindo $l_{k}=\left\{i \mid w_{i}=w_{k}\right\}$. Como mostrado na seção 3, esses modelos inteiros não lineares podem ser reescritos como modelos lineares inteiros.

\subsection{Modelos lineares inteiros}

Lodi e Monaci (2003) apresentaram dois outros modelos lineares para o problema de corte 2-estágios não exato, baseados na restrição de empacotamento das peças em prateleiras (i.e., linhas formando níveis). Cada solução viável do problema é composta de prateleiras. Uma prateleira é uma tira (faixa) da chapa de comprimento $L$, com largura coincidente com a peça de maior largura cortada dela. A peça de maior largura de cada prateleira é dita ser a iniciante da prateleira. Numa solução viável do problema composta de prateleiras, cada item empacotado em uma prateleira pode ser cortado em no máximo 2 estágios (mais aparas).

No primeiro modelo (LM1) de Lodi e Monaci (2003), cada peça é considerada de maneira distinta. Assim, para cada peça tipo $i, i=1, \ldots, m$, definimos $b_{i}$ itens distintos $j$, com $l_{j}=l_{i}, w_{j}=w_{i}$, e $v_{j}=v_{i}$. Denotamos o número total de peças do problema por $n$, ou seja, $n=\sum_{i=1}^{m} b_{i}$, e sem perda de generalidade, admitimos que $w_{1} \geq w_{2} \geq \ldots \geq w_{n}$. As variáveis de decisão do modelo são: $x_{j k}= \begin{cases}1, & \text { se a peça } j \text { é cortada da prateleira } k \\ 0, & \text { caso contrário. }\end{cases}$ $k=1,2, \ldots, m ; j=k, \ldots, n$

Note na definição anterior que $j \geq k$ porque a peça $j$ só pode ser cortada (empacotada) de uma prateleira $k$ cuja largura $\left(w_{k}\right)$ seja no mínimo igual à largura da peça $j\left(w_{j}\right)$, i.e., $w_{k} \geq w_{j}, j \geq k$. No modelo, admite-se que $n$ prateleiras podem ser inicializadas em potencial, cada uma delas tendo como peça iniciante a peça $k, k=1,2, \ldots, n$. Assim, $x_{k k}=1(k=1, \ldots, n)$ implica que a peça $k$ é cortada da prateleira $k$, ou seja, a prateleira $k$ é usada e a peça $k$ é a peça iniciante dessa prateleira. 0 modelo LM1 então é definido por: Modelo LM1

$\max \sum_{j=1}^{n} v_{j} \sum_{k=1}^{j} x_{j k}$

Sujeito a

$\sum_{k=1}^{j} x_{j k} \leq 1$, para $j=1, \ldots, n$

$\sum_{j=k+1}^{n} l_{j} x_{j k} \leq\left(L-l_{k}\right) x_{k k}$, para $k=1, \ldots, n-1$

$\sum_{k=1}^{n} w_{k} x_{k k} \leq W$

com

$x_{j k} \in\{0,1\}, k=1, \ldots, n ; j=k, \ldots, n$

A função objetivo (12) maximiza a soma dos valores das peças cortadas. As restrições (13) impõem que cada peça pode ser cortada no máximo uma vez, e somente de prateleiras cujas larguras sejam no mínimo iguais à sua largura. As restrições (14) impõem que as peças incluídas em cada prateleira não ultrapassem o seu comprimento. A restrição (15) impõe que as prateleiras usadas caibam na largura da chapa. As restrições (16) impõem que as variáveis de decisão sejam binárias. 
No segundo modelo (LM2) de Lodi e Monaci (2003), as peças iguais são consideradas individualmente apenas nas prateleiras, para fins de sua inicialização. Define-se um mapeamento entre tipo de peças $i$, $i=1, \ldots, m$, e prateleiras em potencial, $k, k=1$, $2, \ldots, n$, em que $n$ é igual a $\sum_{i=1}^{m} b_{i}$, como no modelo LM1. Sejam $\alpha_{i}=\sum_{s=1}^{i} b_{s}, i=1, \ldots, m$, e $\alpha_{0}=0$; e $\beta_{k}, k=1,2, \ldots, n$, o tipo de peça que inicializa a prateleira $k$, ou seja, para $k=1,2, \ldots, n, \beta_{k}=i$ sempre que $\alpha_{i-1}+1 \leq k \leq \alpha_{i}$. Observe que se $W_{k}$ é a largura da prateleira $k$, então para $k=1,2, \ldots, n, W_{k}=W_{i}$ sempre que $\alpha_{i-1}+1 \leq k \leq \alpha_{i}$.

Admitindo-se novamente, sem perda de generalidade, que $w_{1} \geq w_{2} \geq \ldots \geq w_{m}$, qualquer peça do tipo $i$ pode ser cortada de uma prateleira $k$, com $k$ inteiro, $1 \leq k \leq \alpha_{i}$, e qualquer prateleira $k$ pode ser utilizada para cortar peças do tipo $j, j$ inteiro, $i \leq j \leq m$, se $\alpha_{i-1}+1 \leq k \leq \alpha_{i}$.

As variáveis de decisão (inteiras) do modelo LM2 são:

$$
x_{i k}=\left\{\begin{array}{l}
\text { número de peças tipo } i \\
\text { cortadas da prateleira } k, \text { se } i \neq \beta_{k} \\
\text { número de peças adicionais do tipo } i \\
\text { cortadas da prateleira } k, \text { se } i=\beta_{k} .
\end{array}\right.
$$

com $i=1,2, \ldots, m ; k=1,2, \ldots, \alpha_{i}$.

0 termo adicional da definição anterior indica que a peça tipo $i$ que inicializa a prateleira é considerada à parte (se a prateleira é inicializada com esse tipo de item). Além dessas variáveis, temos as seguintes variáveis binárias:

$$
q_{k}=\left\{\begin{array}{ll}
1, & \text { se a prateleira } k \text { é usada } \\
0, & \text { caso contrário. }
\end{array} k=1,2, \ldots, n\right.
$$

Sejam $L_{k}$ o comprimento da peça $\beta_{k}$, ou seja, o comprimento da peça que inicializa a faixa $k$, e $W_{k}$ a largura da faixa $k$ que é igual à largura da peça $\beta_{k}$, ou seja, a largura da peça que inicializa a faixa $k$, $k=1, \ldots, n .0$ modelo LM2 é definido por:

\section{Modelo LM2}

$$
\max \sum_{i=1}^{m} v_{i}\left(\sum_{k=1}^{\alpha_{i}} x_{i k}+\sum_{k=\alpha_{i-1}+1}^{\alpha_{i}} q_{k}\right)
$$

Sujeito a

$$
\begin{aligned}
& \sum_{k=1}^{\alpha_{i}} x_{i k}+\sum_{k=\alpha_{i-1}+1}^{\alpha_{i}} q_{k} \leq b_{i}, \text { para } i=1, \ldots, m \\
& \sum_{i=\beta_{k}}^{m} l_{i} x_{i k} \leq\left(L-L_{k}\right) q_{k}, \text { para } k=1, \ldots, n
\end{aligned}
$$

$$
\sum_{k=1}^{n} W_{k} q_{k} \leq W
$$

com

$0 \leq x_{i k} \leq b_{i}$, inteiro, $i=1, \ldots, m ; k=1, \ldots, \alpha_{i}$

$q_{k} \in\{0,1\}, k=1, \ldots, n$

A função objetivo (17) maximiza a soma dos valores das peças cortadas. As restrições (18) impõem que no máximo pode-se cortar a demanda de cada tipo de peça, e que essas peças somente podem ser cortadas de prateleiras cujas larguras sejam no mínimo iguais à largura da peça. As restrições (19) impõem que as peças incluídas em cada prateleira não ultrapassem o comprimento da chapa. A restrição (20) impõe que as prateleiras usadas caibam na largura da chapa. As restrições (21) impõem integralidade das variáveis $x_{i k}$, e, além disso, impõem que nas prateleiras de largura maior ou igual à largura de uma peça, o número de vezes que essa peça pode ser cortada é no máximo a sua demanda. As restrições (22) impõem que as variáveis $q_{k}$ sejam binárias.

Em adição às restrições (18)-(22), Lodi e Monaci (2003) incluem as seguintes restrições redundantes para fortalecer o modelo $\mathrm{LM} 2$ :

$$
\begin{aligned}
& \sum_{s=k}^{\alpha_{i}} x_{i s} \leq b_{i}-\left(k-\alpha_{i-1}\right), \\
& \text { para } i=1, \ldots, m ; k=\alpha_{i-1}+1, \ldots, \alpha_{i}
\end{aligned}
$$

Lodi e Monaci mostraram que os modelos LM1 e LM2 são competitivos comparados com o algoritmo exato de Hifi e Roucairol (2001), utilizando um método de resolução do tipo branch-and-bound do CPLEX, com a adição de algumas desigualdades lineares para evitar simetrias.

Para o modelo LM1, as seguintes desigualdades evitam algumas simetrias:

$x_{t t} \geq x_{t+1, t+1}$ para $i=1, . ., m$;

$t=\alpha_{i-1}+1, \alpha_{i-1}+2, \ldots, \alpha_{i}-1$

$$
\begin{aligned}
& \sum_{s=t+1}^{\alpha_{i}} X_{s t} \geq \sum_{s=t+2}^{\alpha_{i}} X_{s, t+1} \\
& \text { para } i=1, . ., m ; t=\alpha_{i-1}+2, \ldots, \alpha_{i}-1
\end{aligned}
$$

De maneira equivalente, para o modelo LM2, as seguintes desigualdades evitam algumas simetrias:

$q_{t} \geq q_{t+1}$ para $i=1, \ldots, m$;

$t=\alpha_{i-1}+1, \alpha_{i-1}+2, \ldots, \alpha_{i}-1$

$x_{i t} \geq x_{i, t+1}$ para $i=1, \ldots, m$;

$t=\alpha_{i-1}+1, \alpha_{i-1}+2, \ldots, \alpha_{i}-1$ 
Os modelos LM1 e LM2 podem ser estendidos facilmente para o caso exato impondo (ou eliminando) que $x_{j k}=0$ se $w_{k} \neq w_{j}$ no modelo LM1, e $W_{k} \neq w_{j}$ no modelo LM2.

\section{Modelos lineares inteiros alternativos}

\subsection{Modelo linear inteiro 1}

O modelo VAG2 (6)-(11) apresentado na seção 2.1 pode ser linearizado da seguinte forma (HARJUNKOSKl et al., 1997). Seja $\mu_{k p}=\sum_{s=1}^{s_{k}} 2^{s-1} \beta_{k p s}$, em que $\beta_{k p s} \in\{0,1\}$ e $s_{k}$ é tal que: $2^{s_{k}-1} \leq\left\lfloor W / w_{k}\right\rfloor<2^{s_{k}}$, ou seja, $s_{k}$ é o número mínimo de bits necessário para a representação binária de $\mu_{k p}$ (o mesmo poderia ser feito escolhendo-se $\lambda_{k p}^{i}$ ao invés de $\mu_{k p}$; entretanto, o número de variáveis seria maior, na maioria dos casos). Com isso, a restrição não linear (9) pode ser reescrita como:

$\alpha_{i}=\sum_{k=1}^{K} \sum_{p=1}^{P_{k}} \delta_{k}^{i} \lambda_{k p}^{i} \sum_{s=1}^{s_{k}} 2^{s-1} \beta_{k p s}=$

$\sum_{k=1}^{K} \sum_{p=1}^{P_{k}} \delta_{k}^{i} \sum_{s=1}^{s_{k}} 2^{s-1}\left(\lambda_{k p}^{i} \beta_{k p s}\right)$, para $i=1, \ldots, m$

que, por sua vez, pode ser substituída pelo seguinte conjunto de restrições lineares:

$\alpha_{i}=\sum_{k=1}^{K} \sum_{p=1}^{P_{k}} \delta_{k}^{i} \sum_{s=1}^{s_{k}} 2^{s-1} f_{k p s}^{i}$, para $i=1, \ldots, m$

$f_{k p s}^{i} \leq \lambda_{k p}^{i}, \operatorname{para} k=1, \ldots, K, p \in P_{K}, i \in I_{k}, s=1, \ldots, s_{k}$

$f_{k p s}^{i} \geq \lambda_{k p}^{i}-M\left(1-\beta_{k p s}\right)$,

para $k=1, \ldots, K, p \in P_{k}, i \in I_{k}, s=1, \ldots, s_{k}$

$f_{k p s}^{i} \leq M \beta_{k p s}, \operatorname{para} k=1, \ldots, K, p \in P_{k} i \in I_{k}, s=1, \ldots, s_{k}$

$\beta_{k p s} \in\{0,1\}, f_{k p s}^{i} \geq 0$,

para $i=1, \ldots, m, k=1, \ldots, K, p \in P_{k}, s=1, \ldots, s_{k}$

em que $M$ é um número suficientemente grande (e.g., $\left\lfloor L / I_{\min }\right\rfloor$ ). Observe que se $\beta_{k p s}=1$, então corretamente segue que $f_{k p s}^{i}=\lambda_{k p}^{i}$; por outro lado, se $\beta_{k p s}=0$, então corretamente segue que $f_{k p s}^{i}=0$. 0 modelo (6)-(11) é reescrito como:

\section{Modelo 1:}

$\max \sum_{i=1}^{m} v_{i} \alpha_{i}$
Sujeito a

$\sum_{i \in l_{k}} l_{i} \lambda_{k p}^{i} \leq L$, para $k=1, \ldots, K, p \in P_{k}$

$\sum_{k=1}^{K} W_{k} \sum_{p=1}^{P_{k}} \sum_{s=1}^{s_{k}} 2^{s-1} \beta_{k p s} \leq W$

$\alpha_{i}=\sum_{k=1}^{K} \sum_{p=1}^{P_{k}} \delta_{k}^{i} \sum_{s=1}^{s_{k}} 2^{s-1} f_{k p s}^{i}$, para $i=1, \ldots, m$

$f_{k p s}^{i} \leq \lambda_{k p}^{i}, \operatorname{para} k=1, \ldots, K, p \in P_{k}, i \in I_{k}, s=1, \ldots, s_{k}$

$f_{k p s}^{i} \geq \lambda_{k p}^{i}-M\left(1-\beta_{k p s}\right)$,

para $k=1, \ldots, K, p \in P_{k}, i \in I_{k}, s=1, \ldots, s_{K}$

$f_{k p s}^{i} \leq M \beta_{k p s}$, para $k=1, \ldots, K$,

$p \in P_{k}, i \in I_{k}, s=1, \ldots, s_{k}$

$\alpha_{i} \leq b_{i}$, para $i=1, \ldots, m$

com

$\beta_{k p s c}\{0,1\}, \lambda_{k p}^{i} \geq 0$, inteiro, $f_{k p s}^{i} \geq 0, i=1, \ldots, m$;

$k=1, \ldots, K ; p=1, \ldots, P_{k}, s=1, \ldots, s_{k}$

Similarmente ao comentado na seção 2.1, o caso exato pode ser tratado pelo modelo 1 simplesmente redefinindo $l_{k}=\left\{i \mid w_{i}=w_{k}\right\}$.

É possível adicionar ao modelo 1 algumas desigualdades lineares para evitar simetrias:

$$
\sum_{i \in I_{k}} v_{i} \lambda_{k s}^{i} \geq \sum_{i \in I_{k}} v_{i} \lambda_{k, s+1}^{i} \text { para } s=1,2, \ldots, P_{k}-1
$$

Essas desigualdades apenas ordenam as tiras de mesma largura em um padrão, de forma não crescente de sua contribuição para a função objetivo.

A linearização anterior foi também explorada em Yanasse e Morabito $(2006,2008)$ para desenvolver modelos lineares inteiros (de formulações inteiras não lineares), para gerar padrões de corte guilhotinados p-grupo. Observamos que outras transformações 0-1 para as variáveis $\lambda_{j}$ poderiam ser consideradas. Por exemplo, a transformação sugerida em Johnston e Sadinlija (2004) é interessante no caso em que existem valores específicos de $\lambda_{j}$ que podem ser descartados a priori e o número de valores que $\lambda_{j}$ pode assumir não é grande. lsto porque a transformação é pseudopolinomial, portanto, o número de variáveis 0-1 cresce mais rapidamente que a transformação apresentada anteriormente.

\subsection{Modelo linear inteiro 2}

Scheithauer (2002) apresentou um modelo linear inteiro para o problema de geração de padrões 
1-grupo não exatos, que pode ser estendido para gerar padrões guilhotinados 2-estágios não exatos. Por conveniência, apresentamos a seguir o modelo mencionado de Scheithauer. Sejam os parâmetros:

- $P, Q \rightarrow$ número máximo de tiras da esquerda para a direita e do fundo para o topo, respectivamente, no padrão $\left(P=\left\lfloor L / I_{\min }\right\rfloor, Q=\left\lfloor W / w_{\min }\right\rfloor\right)$

e as variáveis de decisão:

- $L_{j} \rightarrow$ comprimento da $j$-ésima tira (da esquerda para a direita) no padrão $(j=1, \ldots, P)$

- $W_{k} \rightarrow$ largura da $k$-ésima tira (do fundo ao topo) no padrão $(k=1, \ldots, Q)$

$x_{i j k}= \begin{cases}1, & \text { se a peça tipo } i \text { é colocada no retângulo } L_{j} \times W_{k} \\ 0, & \text { caso contrário. }\end{cases}$

$\max \sum_{i=1}^{m} \sum_{j=1}^{P} \sum_{k=1}^{Q} v_{i} x_{i j k}$

Sujeito a

$$
\sum_{j=1}^{P} L_{j} \leq L
$$

$$
\sum_{k=1}^{Q} W_{k} \leq W
$$

$\sum_{i=1}^{m} x_{i j k} \leq 1$, para todo $j, k$

$\sum_{i=1}^{m} l_{i} x_{i j k} \leq L_{j}$, para todo $j, k$

$\sum_{i=1}^{m} w_{i} x_{i j k} \leq W_{k}$, para todo $j, k$

$\sum_{j=1}^{P} \sum_{k=1}^{Q} x_{i j k} \leq b_{i}$, para todo $i$

$L_{j} \geq L_{j+1}$, paratodo $i$

$W_{k} \geq W_{k+1}$, para todo $k$

com

$x_{i j k} \in\{0,1\}, L_{j}, W_{k} \geq 0, i=1, \ldots, m ;$

$j=1, \ldots, P ; k=1, \ldots, Q$

A função objetivo (38) maximiza o valor total das peças cortadas no padrão. As restrições (39) e (40) garantem que os comprimentos e larguras das tiras do padrão não excedam o comprimento e a largura da chapa, respectivamente. As restrições (41) impõem que no máximo uma peça é colocada em cada retângulo $L_{j} \times W_{k}$. As restrições (42) e (43) garantem que o comprimento e a largura das peças em cada retângulo $L_{j} \times W_{k}$, não excedam o seu comprimento e a sua largura, respectivamente. As restrições (44) referem-se à disponibilidade de peças e as restrições (47) referem-se à não negatividade e integralidade das variáveis. Observe que as restrições (45) e (46) são adicionadas para eliminar simetrias (e reduzir o espaço de busca). Observe também em (47) que as variáveis $L_{j}$ e $W_{k}$ não precisam ser inteiras.

0 modelo (38)-(47) pode ser estendido para modelar o caso de padrões 2-estágios não exatos, redefinindo-se as variáveis $x_{i j k}$, que agora indicam se a peça tipo $i$ é colocada na $j$-ésima posição da tira $k$ (ao invés do retângulo $L_{j} \times W_{k}$ ).

\section{Modelo 2:}

$\max \sum_{i=1}^{m} \sum_{j=1}^{P} \sum_{k=1}^{Q} v_{i} x_{i j k}$

$\sum_{k=1}^{Q} W_{k} \leq W$

$\sum_{i=1}^{m} x_{i j k} \leq 1$, para todo $j, k$

$\sum_{i=1}^{m} \sum_{j=1}^{P} I_{i} x_{i j k} \leq L$, paratodo $k$

$\sum_{i=1}^{m} w_{i} x_{i j k} \leq W_{k}$, para todo $j, k$

$\sum_{j=1}^{J} \sum_{k=1}^{Q} x_{i j k} \leq b_{i}$, para tado $i$

$W_{k} \geq W_{k+1}$, para todo $k$

com

$x_{i j k} \in\{0,1\}, W_{k} \geq 0, i=1, \ldots, m ;$

$j=1, \ldots, P ; k=1, \ldots, Q$

Para adaptar o modelo 2 para o caso exato, poderíamos pensar que seria suficiente substituir a desigualdade (52) pela igualdade:

$$
\sum_{i=1}^{m} w_{i} x_{i j k}=W_{k} \text {, para todo } j, k
$$

No entanto, essa restrição é válida apenas no caso em que existe alguma peça do tipo $i$ colocada na $j$-ésima posição da tira $k$. Se não existe nenhuma peça nessa posição, então essa restrição não precisa ser satisfeita. Para impor a restrição (56) apenas quando existe alguma peça do tipo $i$ colocada na $j$-ésima posição da tira $k$, introduzimos as seguintes restrições:

$$
\sum_{i=1}^{m} w_{i} x_{i j k} \leq W_{k}+M\left(1-\sum_{i=1}^{m} x_{i j k}\right), \text { para todo } j, k
$$




$$
W_{k} \leq \sum_{i=1}^{m} w_{i} x_{i j k}+M\left(1-\sum_{i=1}^{m} x_{i j k}\right), \text { para todo } j, k
$$

em que $M$ é um número suficientemente grande (e.g., $M=W$ ). Dessa forma, o modelo 2-estágios para o caso exato é obtido simplesmente substituindo as restrições (52) pelas restrições (57) e (58) no modelo 2.

\subsection{Modelo linear inteiro 3}

Um outro modelo linear pode ser proposto a partir do modelo 2, definindo-se variáveis inteiras $x_{i k}$ para 0 número de peças tipo $i$ na tira $k$, baseadas nas variáveis $x_{i j k}$ do modelo 2 , assim como variáveis binárias $y_{i k}$ indicando se uma peça tipo $i$ é alocada na tira $k$.

Variáveis:

- $W_{k} \rightarrow$ largura da $k$-ésima tira (do fundo ao topo) no padrão $(k=1, \ldots, Q)$

$x_{i k}=\sum_{j=1}^{P} x_{i j k}$, ou seja, o número de peças tipo $i$ na tira $k$,

$y_{i k}= \begin{cases}1, & \text { se existe ao menos uma peça tipo } i \\ & \text { colocada na tira } k \\ 0, & \text { caso contrário. }\end{cases}$

0 modelo é dado por:

Modelo 3:

$$
\begin{aligned}
& \max \sum_{i=1}^{m} \sum_{k=1}^{Q} v_{i} x_{i k} \\
& \sum_{i=1}^{m} l_{i} x_{i k} \leq L, \text { para todo } k \\
& W_{i} y_{i k} \leq W_{k}, \text { para todo } i, k \\
& \sum_{k=1}^{Q} W_{k} \leq W
\end{aligned}
$$$$
\sum_{k=1}^{Q} x_{i k} \leq b_{i}, \text { paratodo } i
$$

$x_{i k} \leq M y_{i k}$, paratodo $i, k$

$y_{i k} \leq x_{i k}$, paratodo $i, k$

$W_{k} \geq W_{k+1}$, para todo $k$

com

$x_{i k} \geq 0$, inteiro, $y_{i k} \in\{0,1\}, W_{k} \geq 0$,

$i=1, \ldots, m ; k=1, \ldots, Q$

em que $M$ é um número suficientemente grande (e.g., $M=\left\lfloor L / I_{\min }\right\rfloor$ ). A descrição dessas restrições é similar à das restrições do modelo 2 . Note que a função objetivo (59) e as restrições (60) e (63) são equivalentes a (48), (51) e (53) do modelo 2 , com a substituição das variáveis $X_{i k}=\sum_{j=1}^{P} X_{i j k}$. As restrições (61) garantem que as larguras das peças em cada tira sejam menores ou iguais à largura da tira, similarmente às restrições (52) do modelo 2 , e as restrições (62) e (66) são iguais às restrições (49) e (54) do modelo 2. As restrições (64) garantem que $x_{i k}=0$ se $y_{i k}=0$, e que $y_{i k}=1$ se $x_{i k}>0$. Já as restrições (65) não são necessárias no modelo; elas foram adicionadas apenas para garantir que $y_{i k}=0$ se $x_{i k}=0$. Finalmente as restrições (67) referem-se aos domínios das variáveis; note que $W_{k}$ não precisam ser inteiras, assim como nas restrições (55) do modelo 2.

Similarmente ao modelo 2, para adaptar o modelo 3 para o caso exato, poder-se-ia pensar que seria suficiente substituir a desigualdade (61) pela igualdade:

$w_{i} y_{i k}=W_{k}$, paratodo $i, k$

Novamente, essa restrição é válida apenas se existe ao menos uma peça tipo $i$ colocada na tira $k$. Para impor isso somente quando existe tal peça, introduzimos as seguintes restrições:

$W_{k} \leq w_{i} y_{i k}+M\left(1-y_{i k}\right)$, para todo $i, k$

$w_{i} y_{i k} \leq W_{k}+M\left(1-y_{i k}\right)$, para todo $i, k$

em que $M$ é um número suficientemente grande (e.g., $M=W$ ). Portanto, o modelo para o caso exato 2-estágios é obtido substituindo as restrições (61) pelas restrições (69)-(70) no modelo 3.

Na Tabela 1 os modelos lineares inteiros LM1, LM2, 1, 2 e 3 são comparados em termos de número de variáveis e restrições (sem contabilizar as restrições para evitar simetrias).

Os modelos 1, 2 e 3 foram adaptados de modelos para gerar padrões 1-grupo e podem ser adaptados também para gerar padrões $p$-grupos ( $p \geq 2)$, conforme discutido em Yanasse e Morabito (2006, 2008). De maneira similar, esses modelos podem ser estendidos para gerar também outros padrões simples $n$-estágios com configurações particulares, por exemplo, o padrão 3-estágios com formato T estudado em Cui (2005). Os modelos LM1 e LM2 não podem ser adaptados, pelo menos de maneira simples, para gerar padrões p-grupos.

\section{Experimentos computacionais}

Para efeito de ilustração, apresentamos nesta seção os resultados computacionais obtidos aplicando-se os modelos LM1, LM2, 1, 2 e 3 na resolução de exemplos 
2-estágios. Os resultados a seguir referem-se às versões dos modelos sem inclusões das restrições de simetrias ou para fortalecer os modelos. Observamos que os modelos LM1 e LM2 admitem que os dados de entrada dos itens sejam pré-processados para ordenação das larguras, enquanto os modelos 1 , 2 e 3 não. Todos os modelos foram codificados na linguagem GAMS e resolvidos pelo software de programação linear inteira CPLEX 11 (com as opções padrões default) em um notebook Intel Core 2 com $2 \mathrm{GHz}, 4 \mathrm{~GB}$ RAM e Windows Vista. Para simplificar, na maioria dos exemplos os valores das peças são iguais às suas respectivas áreas; dessa forma, um simples limitante superior igual à área da chapa foi fornecido ao software nos modelos. Exceto pela Tabela 5, os valores apresentados estão em porcentagem da área da chapa utilizada. Em todos os experimentos, admitimos que os cortes do primeiro estágio são paralelos ao comprimento $L$ da chapa, e os cortes do segundo estágio são paralelos à largura $W$ da chapa.

Por ilustração, na Tabela 2 apresentam-se os dados de entrada de um problema simples bidimensional guilhotinado 2-estágios restrito analisado em Vianna et al. (2003). Na Tabela 3 resumimos os resultados computacionais obtidos com os modelos LM1, LM2, 1, 2 e 3 para ambos os casos, exato e não exato. Observe que todos os modelos encontram (e provam) a solução ótima do problema em tempos de execução (em segundos) relativamente pequenos. Na Figura 3 são apresentados os padrões ótimos encontrados pelos modelos para os casos não exato irrestrito (a), não exato restrito (b) e exato restrito (c).

Para analisar o desempenho dos modelos em outros exemplos, geramos aleatoriamente 10 exemplares de problemas 2-estágios restritos, com chapa $(L, W)=(100,100)$ e $m=5$ tipos de peças $\left(l_{i}, w_{i}\right)$, amostrados de distribuições uniformes no intervalo $[0,1 L, 0,5 L]$ e $[0,1 W, 0,5 W]$, respectivamente (depois de amostrado, $l_{i}$ e $w_{i}$ foram simplesmente arredondados).
As quantidades $b_{i}$ também foram amostradas (e depois arredondadas) de distribuições uniformes no intervalo $\left[1,\left\lfloor L / I_{i}\right\rfloor\left\lfloor W / w_{i}\right\rfloor\right]$. Na Tabela 4 os resultados obtidos com os modelos LM1, LM2, 1, 2 e 3 para o caso não exato estão resumidos. Usando o GAMS/CPLEX, todos os modelos encontraram as soluções ótimas de todos os exemplares, mas o modelo LM1 não foi capaz de provar a otimalidade da solução do exemplo 2 (dentro de um limite de tempo imposto de 300 segundos - o número entre parênteses na linha do exemplo 2 corresponde ao gap entre o valor da melhor solução obtida e o valor do limitante superior neste tempo limite) e também teve dificuldades para resolver o exemplo 4. Note que os modelos LM2 e 3 tiveram um desempenho superior aos demais modelos.

A seguir, realizamos alguns experimentos com exemplares bem conhecidos da literatura de problemas restritos de corte e empacotamento com os modelos LM1, LM2, 1, 2 e 3, para obter suas soluções ótimas 2-estágios não exatos (Tabela 5). Os dados de entrada e as soluções (sem restrições de estágios) estão publicados em Christofides e Whitlock (1977) (exemplos CW1, CW2 e CW3 com $m=7,10$ e 20, respectivamente), Wang (1983) (exemplo W com $m=20$ ) e Oliveira e Ferreira (1990) (exemplos OF1 e OF2 com $m=10$ ). Esses exemplos também foram analisados em Viswanathan e Bagchi (1993), Christofides e Hadjiconstantinou (1995), Daza, Alvarenga e Diego (1995), Morabito e Arenales (1996) e Fayard, Hifi e Zissimopoulos (1998). Também utilizamos alguns exemplares irrestritos gcut* da OR-Library analisados em Beasley (1985) e Cintra et al. (2008) (os exemplos gcut1, gcut2 e gcut 3 com $(L, W)=(250,250)$ e $m=10,20,30$, respectivamente, o exemplo gcut12 com $(L, W)=(1000,1000)$ e $m=50$, e o exemplo gcut13 com $(L, W)=(3000$, $3000)$ e $(m=32)$, para verificar o desempenho dos modelos em casos que a demanda $b_{i}$ de peças do tipo $i$ é grande (i.e., $b_{i}=\left\lfloor\left(L / l_{i}\right)\left(W / w_{i}\right)\right\rfloor$ para todo $\left.i\right)$.

Tabela 1. Comparação do número de variáveis e restrições dos modelos LM1, LM2, 1, 2 e 3.

\begin{tabular}{|c|c|c|}
\hline & Número de variáveis & Número de restrições \\
\hline Modelo LM1 & $\sum_{i=1}^{m} b_{i}\left(\sum_{i=1}^{m} b_{1}+1\right) / 2$ & $2 \sum_{i=1}^{m} b_{i}$ \\
\hline Modelo LM2 & $\sum_{i=1}^{m} b_{i}+\sum_{i=1}^{m} \sum_{s=1}^{i} b_{s}$ & $2 \sum_{i=1}^{m} b_{i}+m+1$ \\
\hline Modelo 1 & $m+K \sum_{k=1}^{K} P_{k}+(m+1) K \sum_{k 1}^{K}\left(\left\lceil 1+\log P_{k}\right\rceil\right) P_{k}$ & $2 m+\sum_{k=1}^{K} P_{k}+3 m \sum_{k 1}^{K}\left(\left\lceil 1+\log P_{k}\right\rceil\right) P_{k}$ \\
\hline Modelo 2 & $Q+m P Q$ & $1+2 P Q+2 Q+m$ \\
\hline Modelo 3 & $2 m Q+Q$ & $1+2 Q+3 m Q+m$ \\
\hline
\end{tabular}


Os demais exemplos gcut*w que aparecem na Tabela 5 correspondem aos problemas irrestritos gcut* com os respectivos comprimentos das peças $l_{\text {: }}$ trocados pelas respectivas larguras $w_{i}$, e vice-versa, as larguras $w_{i}$ pelos comprimentos $l_{i}$. Note que isso é equivalente a considerar nos problemas gcut* que os cortes do primeiro estágio são paralelos à largura $W$ da chapa, e os cortes do segundo estágio são paralelos ao comprimento $L$ da chapa. E o exemplo gcut13r é um problema irrestrito com $(L, W)=(3000,3000)$ e $m=64$ tipos de peças (i.e., os 32 tipos de peças do exemplo gcut13, mais os 32 tipos de peças do exemplo gcut13w), ou seja, é equivalente a considerar no problema gcut13 que as peças podem sofrer rotações de $90^{\circ}$.

Observe na Tabela 5 que, usando o GAMS/CPLEX, os modelos LM2 e LM1 tiveram os melhores desempenhos nos problemas restritos, seguidos do modelo 3, modelo 2 e modelo 1 . Nos problemas irrestritos, o modelo LM2 e o modelo 3 em geral tiveram os melhores desempenhos. No caso dos exemplos gcut $13^{*}$, os modelos não foram capazes de encontrar (e provar) a solução ótima dentro do limite de tempo de 300 segundos (exceto para o modelo LM2 no exemplar gcut13w). Assim como na Tabela 4, o número entre parênteses na Tabela 5 corresponde ao gap entre o valor da melhor solução obtida (limitante inferior) e o valor do limitante superior neste tempo limite. Os valores das soluções ótimas dos exemplos gcut 13 e gcut 13 r reportados na literatura são 8906216 e 8997780 , respectivamente (BEASLEY, 1985; CINTRA ET AL., 2008); portanto, os gaps de otimalidade das soluções do modelo LM2 para esses problemas na Tabela 5 são bem pequenos $(0,002 \%$ e $0,03 \%$, respectivamente). Em particular, não

Tabela 2. Exemplo restrito de Vianna et al. (2003) com $(L, W)=(100,100)$ com $m=5$ tipos de peças e $n=22$ peças.

\begin{tabular}{cccc}
\hline Peças $i$ & $l_{i}$ & $w_{i}$ & $b_{i}$ \\
\hline 1 & 33 & 14 & 8 \\
2 & 20 & 38 & 4 \\
3 & 15 & 43 & 4 \\
4 & 40 & 43 & 3 \\
5 & 31 & 44 & 3 \\
\hline
\end{tabular}

Tabela 3. Resultados dos modelos LM1, LM2, 1, 2 e 3 (padrões 2-estágios) para o exemplo de Vianna, Arenales e Gramani (2003).

\begin{tabular}{ccccc}
\multirow{2}{*}{ Exemplo } & \multicolumn{2}{c}{ Caso não exato } & \multicolumn{2}{c}{ Caso exato } \\
\cline { 2 - 5 } & Solução & Tempo & Solução & Tempo \\
\hline LM1 & 98,86 & 0,2 & 91,26 & 0,1 \\
LM2 & 98,86 & 0,2 & 91,26 & 0,1 \\
Modelo 1 & 98,86 & 0,2 & 91,26 & 0,1 \\
Modelo 2 & 98,86 & 0,3 & 91,26 & 0,3 \\
Modelo 3 & 98,86 & 0,3 & 91,26 & 0,2 \\
\hline
\end{tabular}

foi possível resolver o modelo LM1 para o exemplo gcut $13 r$ por falta de memória computacional. Além disso, note que, ao contrário do que se poderia esperar, as soluções obtidas pelos modelos para o gcut13r no tempo limite são piores do que as soluções obtidas pelos mesmos modelos para o gcut $13 \mathrm{w}$ no mesmo tempo limite. Verificou-se que os modelos precisam de mais tempo para obter soluções para o gcut13r tão boas ou melhores do que as obtidas para o gcut13w; por exemplo, o modelo LM2 precisa de mais 100 segundos para obter para o gcut13r a mesma solução obtida para o gcut13w.

As seis últimas linhas da Tabela 5 apresentam resultados adicionais obtidos com versões restritas dos problemas gcut1-3, gcut12-13, limitando-se nesses exemplos as quantidades de peças $b$, de cada tipo $i=1, . ., m$. Os exemplos gcut ${ }^{* a}$ correspondem aos respectivos exemplos gcut* com $b_{i}=1, i=1, . ., m$, enquanto o exemplo gcut $13^{\mathrm{b}}$ corresponde ao exemplo gcut13 com $b_{i}=10$. Note que nesses problemas restritos os modelos LM2 e LM1 novamente tiveram os melhores desempenhos dentro de limite de tempo computacional, seguidos do modelo 3. Convém observar que ao se relaxar o limite de tempo computacional, os modelos LM1, LM2 e 3 não foram capazes de obter a solução ótima para o exemplo gcut $13^{\mathrm{b}}$ devido à falta de memória computacional.

Finalmente, para ilustrar o desempenho dos modelos LM1, LM2, 1, 2 e 3 em um exemplo prático, consideramos um exemplo apresentado em Morabito e Arenales (2000) de uma indústria de móveis. Nas Tabelas 6 e 7 apresentam-se os dados de entrada e os resultados obtidos pelo GAMS/CPLEX para este exemplo

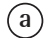

(b)
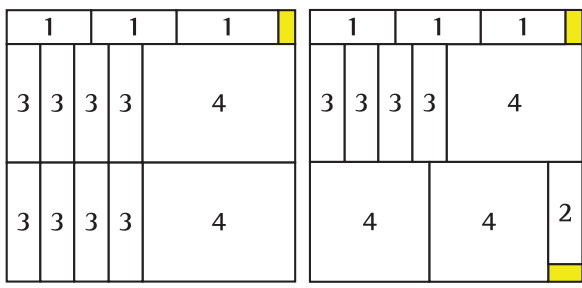

(c)

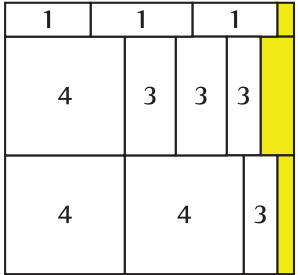

Figura 3. Padrões de corte do exemplo em Vianna et al. (2003): (a) caso não exato irrestrito (valor ótimo $=99,86)$, (b) caso não exato restrito (valor ótimo $=98,86$ ), $($ c) caso exato restrito (valor ótimo $=91,26)$. 
para os casos exato e não exato (os valores das soluções na tabela correspondem às porcentagens das áreas totais das peças cortadas no padrão). Os gaps entre as melhores soluções obtidas (limitantes inferiores) e os limitantes superiores dos modelos no tempo limite de 300 segundos estão apresentados entre parênteses na Tabela 7. No caso não exato, a melhor solução foi obtida pelos modelos LM2, 1 e 3 dentro do limite de tempo (o gap de otimalidade do valor desta solução é menor que $0,3 \%$ ). No caso exato, apenas o modelo 3 foi capaz de provar a otimalidade da solução obtida dentro do limite de tempo. Todos os outros modelos

Tabela 4. Resultados dos modelos LM1, LM2, 1, 2 e 3 (padrões 2-estágios não exato) para os exemplos aleatórios 1-10.

\begin{tabular}{|c|c|c|c|c|c|c|c|c|c|c|}
\hline \multirow[b]{2}{*}{ Exemplo } & \multicolumn{2}{|c|}{ LM1 } & \multicolumn{2}{|c|}{ LM2 } & \multicolumn{2}{|c|}{ Modelo 1} & \multicolumn{2}{|c|}{ Modelo 2} & \multicolumn{2}{|c|}{ Modelo 3} \\
\hline & $\begin{array}{c}\text { Solução } \\
(\%)\end{array}$ & $\begin{array}{c}\text { Tempo } \\
\text { (s) }\end{array}$ & $\begin{array}{c}\text { Solução } \\
(\%)\end{array}$ & $\begin{array}{c}\text { Tempo } \\
\text { (s) }\end{array}$ & $\begin{array}{c}\text { Solução } \\
(\%)\end{array}$ & $\begin{array}{c}\text { Tempo } \\
\text { (s) }\end{array}$ & $\begin{array}{c}\text { Solução } \\
(\%)\end{array}$ & $\begin{array}{c}\text { Tempo } \\
\text { (s) }\end{array}$ & $\begin{array}{c}\text { Solução } \\
(\%)\end{array}$ & $\begin{array}{c}\text { Tempo } \\
\text { (s) }\end{array}$ \\
\hline 1 & 92,72 & 0,4 & 92,72 & 0,2 & 92,72 & 1,2 & 92,72 & 2,1 & 92,72 & 0,1 \\
\hline 2 & $\begin{array}{c}85,99 \\
(2,47 \%)\end{array}$ & $>300$ & 85,99 & 0,3 & 85,99 & 0,8 & 85,99 & 0,8 & 85,99 & 0,2 \\
\hline 3 & 97,00 & 0,4 & 97,00 & 0,1 & 97,00 & 1,1 & 97,00 & 1,5 & 97,00 & 0,1 \\
\hline 4 & 96,02 & 283,7 & 96,02 & 0,1 & 96,02 & 1,0 & 96,02 & 0,2 & 96,02 & 0,3 \\
\hline 5 & 91,02 & 1,5 & 91,02 & 0,2 & 91,02 & 2,5 & 91,02 & 0,3 & 91,02 & 0,3 \\
\hline 6 & 93,06 & 0,2 & 93,06 & 0,1 & 93,06 & 0,6 & 93,06 & 0,2 & 93,06 & 0,1 \\
\hline 7 & 84,14 & 0,7 & 84,14 & 0,2 & 84,14 & 0,2 & 84,14 & 1,2 & 84,14 & 0,2 \\
\hline 8 & 91,76 & 0,3 & 91,76 & 0,1 & 91,76 & 0,3 & 91,76 & 0,7 & 91,76 & 0,2 \\
\hline 9 & 87,36 & 0,2 & 87,36 & 0,1 & 87,36 & 0,1 & 87,36 & 0,1 & 87,36 & 0,1 \\
\hline 10 & 89,46 & 0,2 & 89,46 & 0,1 & 89,46 & 0,2 & 89,46 & 0,2 & 89,46 & 0,3 \\
\hline Média & 90,85 & $31,96^{*}$ & 90,85 & 0,15 & 90,85 & 0,80 & 90,85 & 0,73 & 90,85 & 0,19 \\
\hline
\end{tabular}

*Média de 9 exemplos resolvidos antes do limite de tempo de 300 segundos.

Tabela 5. Resultados dos modelos LM1, LM2, 1, 2 e 3 (padrões 2-estágios não exato) de exemplares da literatura.

\begin{tabular}{|c|c|c|c|c|c|c|c|c|c|c|}
\hline \multirow[b]{2}{*}{ Exemplo } & \multicolumn{2}{|c|}{ LM1 } & \multicolumn{2}{|c|}{ LM2 } & \multicolumn{2}{|c|}{ Modelo 1} & \multicolumn{2}{|c|}{ Modelo 2} & \multicolumn{2}{|c|}{ Modelo 3} \\
\hline & Solução & $\begin{array}{c}\text { Tempo } \\
\text { (s) }\end{array}$ & Solução & $\begin{array}{c}\text { Tempo } \\
\text { (s) }\end{array}$ & Solução & $\begin{array}{c}\text { Tempo } \\
\text { (s) }\end{array}$ & Solução & $\begin{array}{c}\text { Tempo } \\
\text { (s) }\end{array}$ & Solução & $\begin{array}{c}\text { Tempo } \\
\text { (s) }\end{array}$ \\
\hline CW1 & 244 & 0,1 & 244 & 0,1 & 244 & 0,2 & 244 & 0,2 & 244 & 0,2 \\
\hline CW2 & 2535 & 0,3 & 2535 & 0,2 & 2535 & 17,8 & 2535 & 7,5 & 2535 & 4,1 \\
\hline CW3 & 1740 & 0,4 & 1740 & 0,3 & 1740 & 1,7 & 1740 & 0,5 & 1740 & 0,3 \\
\hline W & 2623 & 0,3 & 2623 & 0,2 & 2623 & 3,7 & 2623 & 0,3 & 2623 & 0,7 \\
\hline OF1 & 2713 & 0,1 & 2713 & 0,1 & 2713 & 2,0 & 2713 & 1,8 & 2713 & 0,3 \\
\hline OF2 & 2522 & 0,2 & 2522 & 0,1 & 2522 & 2,7 & 2522 & 1,4 & 2522 & 1,7 \\
\hline gcut 1 & 56460 & 0,2 & 56460 & 0,1 & 56460 & 0,1 & 56460 & 0,1 & 56460 & 0,1 \\
\hline gcut1w & 53808 & 0,2 & 53808 & 0,1 & 53808 & 1,2 & 53808 & 0,1 & 53808 & 0,1 \\
\hline gcut2 & 60076 & 0,6 & 60076 & 0,2 & 60076 & 17,4 & 60076 & 0,5 & 60076 & 0,2 \\
\hline gcut $2 w$ & 60071 & 0,4 & 60071 & 0,2 & 60071 & 2,4 & 60071 & 1,2 & 60071 & 0,3 \\
\hline gcut3 & 60133 & 2,5 & 60133 & 0,4 & 60133 & 186,5 & 60133 & 0,5 & 60133 & 0,4 \\
\hline gcut3w & 60078 & 0,9 & 60078 & 0,4 & 60078 & 290,6 & 60078 & 0,4 & 60078 & 0,6 \\
\hline gcut12 & 977768 & 2,7 & 977768 & 0,6 & 977768 & 38,4 & 977768 & 2,0 & 977768 & 0,8 \\
\hline gcut 12 w & 978776 & 5,5 & 978776 & 0,4 & 978776 & 224,8 & 978776 & 3,7 & 978776 & 0,9 \\
\hline gcut13 & $\begin{array}{c}8798886 \\
(2,29 \%)\end{array}$ & $>300$ & $\begin{array}{c}8906025 \\
(0,77 \%)\end{array}$ & $>300$ & $\begin{array}{c}8865798 \\
(1,51 \%)\end{array}$ & $>300$ & $\begin{array}{c}8780321 \\
(2,50 \%)\end{array}$ & $>300$ & $\begin{array}{c}8873096 \\
(1,43 \%)\end{array}$ & $>300$ \\
\hline gcut13w & $\begin{array}{c}8770548 \\
(2,62 \%)\end{array}$ & $>300$ & 8997780 & 23,2 & $\begin{array}{c}8950588 \\
(0,55 \%)\end{array}$ & $>300$ & $\begin{array}{c}8904528 \\
(1,07 \%)\end{array}$ & $>300$ & $\begin{array}{c}8963660 \\
(0,41 \%)\end{array}$ & $>300$ \\
\hline gcut13r & $* * *$ & & $\begin{array}{c}8994804 \\
(0,06 \%)\end{array}$ & $>300$ & $\begin{array}{c}8628904 \\
(4,30 \%)\end{array}$ & $>300$ & $\begin{array}{c}8964800 \\
(0,39 \%)\end{array}$ & $>300$ & $\begin{array}{c}8941685 \\
(0,65 \%)\end{array}$ & $>300$ \\
\hline gcut $1 w^{a}$ & 48368 & 0,1 & 48368 & 0,1 & 48368 & 0,5 & 48368 & 0,1 & 48368 & 0,1 \\
\hline gcut $2 w^{a}$ & 59307 & 0,1 & 59307 & 0,1 & 59307 & 2,4 & 59307 & 0,3 & 59307 & 0,1 \\
\hline gcut $3 w^{a}$ & 59895 & 0,2 & 59895 & 0,1 & 59895 & 26,9 & 59895 & 0,9 & 59895 & 0,4 \\
\hline gcut 12wa & 970744 & 0,1 & 970744 & 0,1 & 970744 & 93,2 & 970744 & 2,6 & 970744 & 0,8 \\
\hline gcut $13^{\mathrm{a}}$ & 7822230 & 2,5 & 7822230 & 2,4 & $\begin{array}{l}7575276 \\
(18,81 \%)\end{array}$ & $>300$ & $\begin{array}{l}7821149 \\
(15,07 \%)\end{array}$ & $>300$ & 7822230 & 35,1 \\
\hline gcut $13^{b}$ & $\begin{array}{c}8886680 \\
(1,28 \%)\end{array}$ & $>300$ & $\begin{array}{c}8889656 \\
(1,03 \%)\end{array}$ & $>300$ & $\begin{array}{c}8559400 \\
(5,14 \%)\end{array}$ & $>300$ & $\begin{array}{c}8871112 \\
(1,45 \%)\end{array}$ & $>300$ & $\begin{array}{c}8870120 \\
(1,46 \%)\end{array}$ & $>300$ \\
\hline
\end{tabular}

***Falta de memória computacional. 
Tabela 6. Exemplo prático de uma indústria de móveis com $(L, W)=(1850,3670)$ com $n=13$ tipos de peças.

\begin{tabular}{cccccc}
\hline Peças $i$ & $l_{i}$ & $w_{i}$ & Peças $i$ & $l_{i}$ & $w_{i}$ \\
\hline 1 & 609 & 274 & 8 & 705 & 300 \\
2 & 380 & 274 & 9 & 465 & 330 \\
3 & 425 & 330 & 10 & 480 & 330 \\
4 & 650 & 361 & 11 & 674 & 302 \\
5 & 348 & 270 & 12 & 674 & 270 \\
6 & 705 & 270 & 13 & 636 & 270 \\
7 & 718 & 328 & & & \\
\hline
\end{tabular}

Tabela 7. Resultados dos modelos LM1, LM2, 1, 2 e 3 para o exemplo de uma indústria de móveis

\begin{tabular}{|c|c|c|c|c|c|c|c|c|c|c|}
\hline \multirow{2}{*}{ Exemplo } & \multicolumn{2}{|c|}{ LM1 } & \multicolumn{2}{|c|}{ LM2 } & \multicolumn{2}{|c|}{ Modelo 1} & \multicolumn{2}{|c|}{ Modelo 2} & \multicolumn{2}{|c|}{ Modelo 3} \\
\hline & Solução & Tempo (s) & Solução & Tempo (s) & Solução & Tempo (s) & Solução & Tempo (s) & Solução & Tempo (s) \\
\hline Não exato & $\begin{array}{c}98,32 \\
(1,71 \%)\end{array}$ & $>300$ & $\begin{array}{c}99,18 \\
(0,83 \%)\end{array}$ & $>300$ & $\begin{array}{c}99,18 \\
(0,83 \%)\end{array}$ & $>300$ & $\begin{array}{c}98,74 \\
(1,28 \%)\end{array}$ & $>300$ & $\begin{array}{c}99,18 \\
(0,83 \%)\end{array}$ & $>300$ \\
\hline Exato & $\begin{array}{c}98,34 \\
(1,69 \%)\end{array}$ & $>300$ & $\begin{array}{c}98,91 \\
(0,62 \%)\end{array}$ & $>300$ & $\begin{array}{c}98,91 \\
(1,10 \%)\end{array}$ & $>300$ & $\begin{array}{c}98,91 \\
(1,10 \%)\end{array}$ & $>300$ & 98,91 & 31,1 \\
\hline
\end{tabular}

também encontraram essa mesma solução dentro do limite de tempo, exceto o modelo LM1 (que necessitou de mais de 600 segundos para encontrá-la).

\section{Considerações finais}

Neste estudo revemos modelos existentes e propomos novos modelos para gerar padrões guilhotinados restritos 2-estágios bidimensionais, incluindo os casos exato e não exato. Esses modelos são úteis para a pesquisa e desenvolvimento de métodos de solução mais eficientes, explorando características específicas e estruturas particulares, decomposição do modelo, relaxações do modelo etc. Tais modelos também são úteis para avaliar o desempenho de heurísticas, pois eles permitem (ao menos para problemas de tamanho moderado) estimar o gap de otimalidade de soluções heurísticas.

Uma comparação mais efetiva dos desempenhos dos modelos está além do escopo deste trabalho. No entanto, para ilustrar a aplicação dos modelos, apresentamos resultados de alguns experimentos computacionais usando um software comercial bem conhecido, a linguagem de modelagem GAMS e o otimizador CPLEX. Esses resultados mostraram que os esforços computacionais para solucionar os modelos podem ser bem diferentes. 0 modelo 3 (proposto neste estudo) obteve melhor desempenho que o modelo 1 (linearização proposta neste estudo do modelo não linear de Vianna, Arenales e Gramani (2003)) e o modelo 2 (extensão do modelo linear de SCHEITHAUER, 2002). Além disso, os modelos 1, 2 e 3 foram competitivos com os modelos LM1 e LM2 propostos em Lodi e Monaci (2003) nos experimentos aqui realizados, principalmente o modelo 3 .
Cabe observar que para o caso em que a orientação das peças não está fixa é suficiente adicionar mais peças aos modelos 1 , 2 e 3 correspondentes às peças originais rotacionadas em $90^{\circ}$. A restrição na limitação do número de peças é ajustada de acordo, isto é, limitamos a soma das peças rotacionadas mais as peças em sua orientação original. A extensão dos modelos 1, 2 e 3 para tratar problemas de corte bidimensionais guilhotinados restritos com mais do que 2 estágios é um tópico para pesquisa futura. Outra linha de pesquisa interessante seria estudar limitantes inferiores e superiores efetivos, restrições de simetria e desigualdades válidas específicas para os modelos 1,2 e 3 , com vistas a reduzir os tempos de execução necessários para resolvê-los.

Um comentário final é que para resolvermos o problema de corte de estoque com padrões bidimensionais 2-estágios, usando a abordagem de Gilmore e Gomory (1965) com geração de colunas, precisamos gerar um padrão de corte 2-estágios restrito a cada iteração do método simplex, isto é, resolver um problema da mochila bidimensional com 2 estágios. Assim, é grande o interesse em se ter um método eficiente de resolução do problema de geração destes padrões de cortes. Com os modelos 1 , 2 e 3 , pudemos resolver exemplares deste problema da mochila bidimensional com vários tipos de itens em poucos segundos. Além disso, notamos que em vários exemplares os tempos computacionais para se obter uma solução ótima são bem menores do que os tempos computacionais totais para se provar a otimalidade dessa solução. Uma perspectiva interessante para pesquisa futura seria explorar isso num procedimento de geração de colunas baseado nos modelos 1, 2 e 3 dentro da abordagem de Gilmore e Gomory, e comparar os resultados obtidos com 
outras abordagens conhecidas da literatura, como por exemplo as recentemente propostas em Macedo, Alves e Carvalho (2010) e Silva, Alvelos e Carvalho (2010).

\section{Referências}

ALEM, D. J.; MORABITO, R. Production planning in furniture settings via robust optimization. Computers \& Operations Research, v. 39, n. 2, p. 139-150, 2012. http://dx.doi. org/10.1016/j.cor.2011.02.022

ARENALES, M.; MORABITO, R.; YANASSE, H. (Eds.). Special issue: Cutting and packing problems. Pesquisa Operacional, v. 19, n. 2, p. 107-299, 1999.

BEASLEY, J. Algorithms for unconstrained two-dimensional guillotine cutting. Journal of the Operational Research Society, v. 36, p. 297-306, 1985.

BELOV, G.; SCHEITHAUER, G. A branch-and-bound-andprice algorithm for one-dimensional stock cutting and two-dimensional two-stage cutting. European Journal of Operational Research, v. 171, p. 85-106, 2006. http:// dx.doi.org/10.1016/j.ejor.2004.08.036

BISCHOFF, E.; WAESCHER, G. (Eds.). Special issue: Cutting and packing. European Journal of Operational Research, v. 84 , n. 3, 1995. http://dx.doi.org/10.1016/03772217(95)00018-1

CARNIERI,C.;GUILLERMO,A.;GAVINHO,L.Solutionprocedures for cutting lumber into furniture parts. European Journal of Operational Research, v. 73, p. 495-501, 1994. http:// dx.doi.org/10.1016/0377-2217(94)90244-5

CHRISTOFIDES, N.; HADJICONSTANTINOU, E. An exact algorithm for orthogonal 2-D cutting problems using guillotine cuts. European Journal of Operational Research, v. 83, p. 21-38, 1995. http://dx.doi.org/10.1016/03772217(93)E0277-5

CHRISTOFIDES, N.; WHITLOCK, C. An algorithm for twodimensional cutting problems. Operations Research, v. 25 , n. 1 , p. $30-44,1977$. http://dx.doi.org/10.1287/ opre.25.1.30

CINTRA, G. F. et al. Algorithms for two-dimensional cutting stock and strip packing problems using dynamic programming and column generation. European Journal of Operational Research, v. 191, p. 61-85, 2008. http:// dx.doi.org/10.1016/j.ejor.2007.08.007

CUl, Y. An exact algorithm for generating homogeneous T-shape cutting patterns. Computers \& Operations Research, v. 32, p. 143-152, 2005. http://dx.doi. org/10.1016/S0305-0548(03)00208-9

DAZA, V. P.; ALVARENGA, A. G.; DIEGO, J. Exact solutions for constrained two-dimensional cutting problems. European Journal of Operational Research, v. 84, p. 633-644, 1995. http://dx.doi.org/10.1016/03772217(95)00028-0

DOWSLAND, K.; DOWSLAND, W. Packing problems. European Journal of Operational Research, v. 56, p. 2-14, 1992 http://dx.doi.org/10.1016/0377-2217(92)90288-K

DYCKHOFF, H. A new linear programming approach to the cutting stock problem. Operations Research, v. 29, n. 6, p. 1092-1104, 1981. http://dx.doi.org/10.1287/ opre.29.6.1092

DYCKHOFF, H.; FINKE, U. Cutting and packing in production and distribution: Typology and bibliography.
Heidelberg: Springler-Verlag Co., 1992. http://dx.doi. org/10.1007/978-3-642-58165-6

DYCKHOFF, H.; SCHEITHAUER, G.; TERNO, J. Cutting and packing. In: AMICO, M.; MAFFIOLI, F.; MARTELLO, S. (Eds.). Annotated bibliographies in combinatorial optimisation. New York: John Wiley \& Sons, 1997. p. 393-414.

DYCKHOFF, H.; WAESCHER, G. (Eds.). Special issue: Cutting and packing. European Journal of Operational Research, v. 44, n. 2, 1990.

EURO SPECIAL INTEREST GROUP ON CUTTING AND PACKING - ESICUP. Apdio, 2011. Disponível em: <http:// www.apdio.pt/sicup/>.

FARLEY, A. Practical adaptations of the Gilmore-Gomory approach to cutting stock problems. OR Spektrum, v. 10, p. 113-123, 1983. http://dx.doi.org/10.1007/ BF01720210

FAYARD, D.; HIFl, M.; ZISSIMOPOULOS, V. An efficient approach for large-scale two-dimensional guillotine cutting stock problems. Journal of the Operational Research Society, v. 49, p. 1270-1277, 1998.

FORONDA, S.; CARINO, H. A heuristic approach to the lumber allocation and manufacturing in hardwood dimension and furniture manufacturing. European Journal of Operational Research, v. 54, p. 151-162, 1991. http:// dx.doi.org/10.1016/0377-2217(91)90294-6

GILMORE, P.; GOMORY, R. Multistage cutting stock problems of two and more dimensions. Operations Research, v. 14, p. 94-120, 1965. http://dx.doi.org/10.1287/opre.13.1.94

GRAMANI, M.; FRANÇA, P. The combined cutting stock and lot-sizing problem in industrial processes. European Journal of Operational Research, v. 174, n. 1, p. 509521, 2006. http://dx.doi.org/10.1016/j.ejor.2004.12.019

GRAMANI, M. C. N. ; FRANÇA, P. M.; ARENALES, M. N. A lagrangean relaxation approach to a coupled lot-sizing and cutting stock problem. International Journal of Production Economics, v. 119, p. 219-227, 2009. http:// dx.doi.org/10.1016/j.ijpe.2009.02.011

HARJUNKOSKI, 1. et al. Different strategies for solving bilinear integer non-linear programming problems with convex transformations. Computers \& Chemical Engineering, v. 21, p. 487-492, 1997.

HIFl, M. The DH/KD algorithm: A hybrid approach for unconstrained two-dimensional cutting problems. European Journal of Operational Research, v. 97, n. 1, p. 41-52, 1997. http://dx.doi.org/10.1016/S03772217(96)00060-4

$\mathrm{HIFl,} \mathrm{M.} \mathrm{(Ed.).} \mathrm{Special} \mathrm{issue} \mathrm{on} \mathrm{cutting} \mathrm{and} \mathrm{packing.} \mathrm{Studia}$ Informatica Universalis, v. 2, p. 1-161, 2002.

$\mathrm{HIFl}$, M.; ROUCAIROL, C. Approximate and exact algorithms for constrained (un)weighted two-dimensional twostaged cutting stock problems. Journal of Combinatorial Optimization, v. 5, p. 465-494, 2001. http://dx.doi. org/10.1023/A:1011628809603

HINXMAN, A. The trim-loss and assortment problems: A survey. European Journal of Operational Research, v. 5, p. $8-18$, 1980. http://dx.doi.org/10.1016/03772217(80)90068-5

JOHNSTON, R. E.; SADINLIJA, E. A new model for complete solutions to one-dimensional cutting stock problems. European Journal of Operational Research, v. 153, p. 176-183, 2004. http://dx.doi.org/10.1016/S03772217(02)00704-X 
KENDALL, G.; DANIELS, K.; BURKE, E. K. Special issue: Cutting, packing, layout and space allocation. Annals of Operations Research, v. 179, n. 1, 2010.

LIROV, Y. (Ed.). Special issue: Cutting stock: Geometric resource allocation. Mathematical and Computer Modelling, v. 16, n. 1, 1992.

LODI, A.; MARTELLO, S.; MONACl, M. Two-dimensional packing problems: a survey. European Journal of Operational Research, v. 141, p. 241-252, 2002. http:// dx.doi.org/10.1016/S0377-2217(02)00123-6

LODI, A.; MONACl, M. Integer programming models for 2-staged two-dimensional knapack problems. Mathematical Programming, v. 94, p. 257-278, 2003. http://dx.doi.org/10.1007/s10107-002-0319-9

MACEDO, R.; ALVES, C.; CARVALHO, J. M. V. Arc-flow model for two-dimensional guillotine cutting stock problem. Computers \& Operations Research, v. 37, p. 9911001, 2010. http://dx.doi.org/10.1016/j.cor.2009.08.005

MARTELLO, S. (Ed.). Special issue: Knapsack, packing and cutting, Part 1: One-dimensional knapsack problems. INFOR, v. 32, n. 3, 1994a.

MARTELLO, S. (Ed.). Special issue: Knapsack, packing and cutting, Part 11: Multidimensional knapsack and cutting stock problems. INFOR, v. 32, n. 4, 1994b.

MORABITO, R.; ARENALES, M. Staged and constrained two-dimensional guillotine cutting problems: An and/ or-graph approach. European Journal of Operational Research, v. 94, p. 548-560, 1996. http://dx.doi. org/10.1016/0377-2217(95)00128-X

MORABITO, R.; ARENALES, M. Optimizing the cutting of stock plates in a furniture company. International Journal of Production Research, v. 38, n. 12, p. 2725-2742, 2000. http://dx.doi.org/10.1080/002075400411457

MORABITO, R.; BELLUZZO, L. Optimising the cutting of wood fibre plates in the hardboard industry. European Journal of Operational Research, v. 183, p. 1405-1420, 2007. http://dx.doi.org/10.1016/j.ejor.2005.11.066

MORABITO, R.; GARCIA, V. The cutting stock problem in a hardboard industry: A case study. Computers \& Operations Research, v. 25, n. 6, p. 469-485, 1998. http://dx.doi.org/10.1016/S0305-0548(97)00087-7

MORABITO, R.; ARENALES, M. N.; YANASSE, H. H. (Eds.). Special issue on Cutting, packing and related problems. International Transactions in Operations Research, v. 16, n. 6, 2009. http://dx.doi.org/10.1111/j.14753995.2009.00739.x

MORABITO, R.; PUREZA, V. A heuristic approach based on dynamic programming and AND/OR-graph search for the constrained two-dimensional guillotine cutting problem. Annals of Operation Research, v. 179, p. 297-315, 2010. http://dx.doi.org/10.1007/s10479-008-0457-4

MUKHACHEVA, E. A. (Ed.). Decision making under conditions of uncertainty: cutting -packing problems. Ufa: The International Scientific Collection, 1997.

OLIVEIRA, J. F.; FERREIRA, J. S. An improved version of Wang's algorithm for two-dimensional cutting problems. European Journal of Operational Research, v. 44, p. 256-266, 1990. http://dx.doi.org/10.1016/03772217(90)90361-E

POLDI, K. C.; ARENALES, M. N. Heurísticas para o problema de corte de estoque unidimensional inteiro. Pesquisa Operacional, v. 26, p. 473-492, 2006. http://dx.doi. org/10.1590/S0101-74382006000300003
POLDI, K. C.; ARENALES, M. N. Heuristics for the onedimensional cutting stock problem with limited multiple stock lengths. Computers \& Operations Research, v. 36, p. 2074-2081, 2009. http://dx.doi.org/10.1016/j. cor.2008.07.001

RANGEL, S.; FIGUEIREDO, A. G.; ALTAMIRO, G. 0 problema de corte de estoque em indústrias de móveis de pequeno e médio portes. Pesquisa Operacional, v. 28, p. 451-472, 2008. http://dx.doi.org/10.1590/S010174382008000300004

RIEHME, J.; SCHEITHAUER, G.; TERNO, J. The solution of two-stage guillotine cutting stock problems having extremely varying order demands. European Journal of Operational Research, v. 91, p. 543-552, 1996. http:// dx.doi.org/10.1016/0377-2217(95)00200-6

SANTOS, S. G.; ARAUJO, S. A.; RANGEL, M. S. Integrated cutting machine programming and lot sizing in furniture industry. Pesquisa Operacional para o Desenvolvimento, v. 3, p. 249-166, 2011.

SCHEITHAUER, G. On a two-dimensional guillotine cutting problem. In: INTERNATIONAL FEDERATION OF OPERATIONAL RESEARCH SOCIETIES - IFORS, 16., 2002, Edinburgh. Proceedings ... Edinburgh, 2002.

SILVA, E.; ALVELOS, F.; CARVALHO, J. M. V. An integer programming model for two- and three-stage twodimensional cutting stock problems. European Journal of Operational Research, v. 205, p. 699-708, 2010. http:// dx.doi.org/10.1016/j.ejor.2010.01.039

SWEENEY, P.; PATERNOSTER, E. Cutting and packing problems: A categorised, application-oriented research bibliography. Journal of the Operational Research Society, v. 43, p. 691-706, 1992.

VIANNA, A. C. G.; ARENAlES, M. N.; GRAMANI, M. C. N. Two-stage and constrained two-dimensional guillotine cutting problems. São Carlos: USP, 2003. p. 1-28. (Notas - Série Computação, n. 69).

VISWANATHAN, K.; BAGCHI, A. Best-first search methods for constrained two-dimensional cutting stock problems. Operations Research, v. 41, n. 4, p. 768-776, 1993. http://dx.doi.org/10.1287/opre.41.4.768

YANASSE, H. H.; KATSURAYAMA, D. M. Checkerboard patterns: proposals for its generation. International Transactions in Operational Research, v. 12, p. 21-45, 2005. http:// dx.doi.org/10.1111/j.1475-3995.2005.00488.x

YANASSE, H. H.; ZINOBER, A.; HARRIS, R. Two-dimensional cutting stock with multiple stock sizes. Journal of the Operational Research Society, v. 42, n. 8, p. 673683. 1991.

YANASSE, H. H.; MORABITO, R. Linear models for onegroup two-dimensional guillotine cutting problems. International Journal of Production Research, v. 44, n. 17, p. 3471-3491, 2006. http://dx.doi. org/10.1080/00207540500478603

YANASSE, H. H.; MORABITO, R. A note on linear models for two-group and three-group two-dimensional guillotine cutting problems. International Journal of Production Research, v. 46, n. 21, p. 6189-6206, 2008. http://dx.doi. org/10.1080/00207540601011543

WAESCHER, G.; GAU, T. Heuristics for the integer onedimensional cutting stock problem: a computational study. OR Specktrum, v. 18, p. 131-144, 1996. http:// dx.doi.org/10.1007/BF01539705 
WAESCHER, G.; HAUSSNER, H.; SCHUMANN, H. An improved typology of cutting and packing problems. European Journal of Operational Research, v. 183, p. 11091130, 2007. http://dx.doi.org/10.1016/j.ejor.2005.12.047

WANG, P. Two algorithms for constrained two-dimensional cutting stock problems. Operations Research, v. 31, p. 573-586, 1983. http://dx.doi.org/10.1287/ opre.31.3.573

WANG, P.; WAESCHER, G. (Eds.). Special issue on cutting and packing problems. European Journal of Operational
Research, v. 141, p. 239-469, 2002. http://dx.doi. org/10.1016/S0377-2217(02)00122-4

\section{Agradecimentos}

Gostaríamos de agradecer a um dos revisores anônimos pelos úteis comentários e sugestões. Esta pesquisa foi parcialmente financiada pelo CNPq e pela Fapesp.

\title{
Linear and nonlinear integer models for constrained two-stage two-dimensional knapsack problems
}

\begin{abstract}
In this work we review some linear and nonlinear integer models to generate two stage two-dimensional guillotine cutting patterns, including the constrained, non constrained, exact and non exact cases. These problems are particular cases of the two dimensional knapsack problems. We also present new models to generate these cutting patterns, based on adaptations and extensions of models that generate one-group constrained two dimensional cutting patterns. Two stage patterns arise in different cutting processes like, for instance, in the furniture industry and wooden hardboards. The models are useful for the research and development of more efficient methods, exploring particular structures, the model decomposition, model relaxations etc. They are also useful to evaluate the performance of heuristics, since they allow (at least for problems of moderate sizes) an estimative of the optimality gap of the solutions obtained by heuristics. To illustrate the application of the models we analyze the results of some computational experiments with instances of the literature and other generated randomly. The results were produced using a known commercial software and they show that the necessary computational effort to solve the models can be very different.
\end{abstract}

\section{Keywords}

Cutting and packing problems. Two-dimensional knapsack. Two-stage guillotine cut. Linear and nonlinear integer models. Furniture industry. 